'Unidad de Neonatología, Maternidad Hospital Clínico Universidad de Chile. 2Programa de Genética Humana. Instituto de Ciencias Biomédicas. Facultad de Medicina, Universidad de Chile. anterna Carrera de Medicina Universidad de Chile.

Recibido el 3 de diciembre de 2014, aceptado el 28 de abril de 2015.

Correspondencia a: Dr. Julio Nazer Herrera jnazer@hcuch.cl

\section{ECLAMC: 41 años de vigilancia de la holoprosencefalia en Chile. Período 1972-2012}

\author{
JULIO NAZER HERRERA ${ }^{1}$, LUCÍA CIFUENTES OVALLE², \\ ANDREA CORTEZ LÓPEZ
}

\section{Frequency of holoprosencephaly in Chile}

Background: Holoprosencephaly is a structural anomaly of the brain that consists in a defect of the prosencephalon development that leads to face and neurological defects of variable intensity. Aim: To estimate holoprosencephaly prevalence at birth. Patients and Methods: All cases of holoprosencephaly, born alive or stillbirths, registered in the 15 Chilean Hospitals of the Latin American Collaborative Study of Congenital Malformations (ECLAMC) between 1972 and 2012, were studied. Craniofacial and other anomalies found in newborns affected by holoprosencephaly are described. Results: Fifty five cases of holoprosencephaly (58\% males) were found among the 798.222 registered births (rendering a prevalence at birth of 0.69 per 10.000 newborns). The most common cranial defect was medial cleft lip with cleft palate (27.3\%), bilateral cleft lip (11\%) or both (38.2\%), cyclopia (14\%), single nostril (10.9\%) and proboscis (9.1\%). Eleven percent cases had a trisomy 13. A slight increase in prevalence over time was observed. Conclusions: Holoprosencephaly has a low frequency in Chile and is associated to trisomy 13. The increase in prevalence could be explained by a better prenatal diagnosis (ultrasonography).

(Rev Med Chile 2015; 143: 874-879)

Key words: Cyclopia; Holoprosencephaly; Nervous system malformations.

\section{L} a Holoprosencefalia (HPE) es una entidad compleja que incluye una gran variedad de anomalías del cráneo y de la cara debida a una alteración del desarrollo del cerebro, que se produce entre la cuarta y octava semana de gestación y que consiste en falta de desarrollo del prosencéfalo o cerebro anterior del embrión ${ }^{1}$. El resultado de esta anomalía del desarrollo es la aparición de alteraciones neurológicas y defectos de la cara de intensidad variable, que van desde la ausencia del frenillo bajo el labio superior hasta la ciclopia.

La prevalencia de esta patología es muy variable desde 1: 250 embriones a 1: 16.000 nacimientos ${ }^{2}$. Esto sugiere que una gran cantidad de casos se eliminan espontáneamente al comienzo de la gestación. La prevalencia al nacimiento se estima entre 0,6 y 0,8 por 10.000 nacimientos. Según un estudio realizado en California, los individuos hispánicos blancos presentan un riesgo aumentado al compararlos con los blancos no hispánicos.

La etiología de la HPE es muy heterogénea y muy variada patogénicamente. Sólo entre 15 y $20 \%$ de todos los casos es posible reconocer las causas, que pueden ser genéticas (monogénicas, cromosómicas) y teratogénicas (ambientales) ${ }^{3}$. En los casos de HPE de causa genética el modo de herencia generalmente es de transmisión autosómica dominante ${ }^{4}$. Menos común es la transmisión recesiva. Se ha identificado al menos 9 genes con mutaciones involucrados en la etiopatogenia de la HPE, entre ellos Sonic Hedgehog (SHH) que es responsable de 3,7\% de todos los casos de HPE y de $17 \%$ de los casos familiares. Berry y cols ${ }^{5}$ en una 
serie de 38 fetos con holoprosencefalia encontró 27 con cariotipo normal (71\%), y 11 con cariotipo anormal (29\%), 73\% de estos últimos eran trisomías 13. Esta asociación ha sido comunicada por otros autores ${ }^{6,7}$.

Esta patología ha sido muy estudiada por numerosos autores, ya en el Siglo XIX se publicaron numerosos trabajos con abundante información sobre la HPE. Cohen ${ }^{1}$ publicó un extenso estudio sobre la teratogénesis en HPE y en él informa que fue Dareste (1891) el primero en reproducir experimentalmente un caso de ciclopia, demostrando la importancia de los teratógenos ambientales.

Numerosos teratógenos son sospechosos de producir HPE en humanos, químicos como el alcohol, quinina, ácido retinoico, compuestos salicilados, difenilhidantoina, cortisona; infecciosos como el citomegalovirus y el virus de la Rubeola ${ }^{8}$. DeMeyer ${ }^{9}$ propuso una clasificación de acuerdo a la gravedad del cuadro clínico:

En primer lugar la HPE alobar, que es la forma más severa. En ella hay una fusión completa de los hemisferios cerebrales y ventrículos laterales. Los hemisferios están fusionados formando un ventrículo único central, con ausencia del cuerpo calloso y de la hoz central. Generalmente, no hay cuernos anteriores o inferiores de los ventrículos laterales y no existe el tercer ventrículo por fusión de los tálamos. El diagnóstico por medio de la ecografía sólo se puede hacer después de la $10^{\mathrm{a}}$ semana de gestación. Es frecuente la asociación con aberraciones cromosómicas.

Le sigue la HPE semilobar. Aquí la fusión de los hemisferios no es total. Sólo existe fusión de la parte anterior del encéfalo, mientras que la parte posterior permanece separada.

La tercera forma es HPE lobar. Es la forma clínica más leve de HPE y se caracteriza por la separación de los hemisferios cerebrales y los ventrículos laterales izquierdo y derecho con algún grado de fusión del neocórtex frontal. Alrededor de 19\% de pacientes con HPE presentan el tipo lobar.

Numerosas son las anomalías craneofaciales que se asocian frecuentemente a la HPE y son tan características que según Demeyer en la HPE "face predicts the brain". Las más frecuentes son ciclopia, los globos oculares y órbitas están total o parcialmente fusionadas con ausencia de las estructuras de la nariz y puede existir una proboscis en la línea media y por encima de los globos oculares, etmocefalia en que hay hipotelorismo ocular extremo, los globos oculares están muy juntos pero separados, con una proboscis entre ellos y cebocefalia en que la nariz es pequeña y aplanada con orificio nasal único, hipotelorismo ocular y ausencia de las estructuras premaxilares y septum nasal. Labio leporino central, hipotelorismo ocular y nariz plana. Por último, alteraciones faciales leves como Incisivo central único, ausencia de frenillo bajo el labio superior, hipotelorismo ocular leve, nariz aplanada. Puede asociarse también a anomalías fuera de la cara, como polidactilia, espina bífida, anencefalia, anomalías cardiovasculares y renales.

El diagnóstico prenatal de la HPE se realiza especialmente a través del estudio del cerebro fetal y de la búsqueda de malformaciones de la cara como ciclopia, paladar hendido central ${ }^{10}$. Este estudio debe hacerse después de la décima semana de gestación ya que hasta la novena semana existe normalmente un ventrículo único en el cerebro anterior. La forma de HPE alobar es la que se puede diagnosticar más fácilmente debido a la mayor severidad de sus anomalías, que se caracterizan por presentar un ventrículo central único sin eco medio, ausencia de los cuernos anteriores, posteriores e inferiores de los ventrículos laterales, ausencia de cuerpo calloso y del septum pellucidum y del tercer ventrículo ${ }^{11,12}$.

La HPE semilobar es más difícil, ya que los hallazgos son similares a la forma alobar. Se puede diferenciar los cuernos posteriores de los ventrículos laterales y a veces se encuentra una separación incompleta de los tálamos con un tercer ventrículo hipotrófico ${ }^{13}$.

El pronóstico de la HPE alobar es malo. Prácticamente es una anomalía incompatible con la vida, la gran mayoría de los casos fallecen a las pocas horas o días del nacimiento. La HPE lobar puede tener expectativas de vida normales, pero debe advertírsele a los padres que van a presentar retardo mental y otras secuelas neurológicas importantes.

Es fundamental conocer el cariotipo del niño sabiendo que muchos de ellos pueden tener aberraciones cromosómicas con un importante riesgo de recurrencia.

En los casos leves de HPE lobar es necesario corregir algunas anomalías, como el Labio leporino y fisura palatina para darle una oportunidad de mejor alimentación.

Los objetivos de este estudio son determinar la real prevalencia de esta patología en Chile, si ésta 
ha tenido algún grado de variabilidad a través del tiempo y que factores pudieran haber influido en ello.

\section{Pacientes y Método}

El ECLAMC (Estudio Colaborativo Latino Americano de Malformaciones Congénitas) fue creado en el año 1967 como un Programa de vigilancia epidemiológica de estas patologías. Chile ingresó en septiembre de 1969. Su objetivo principal es la de monitorizar las frecuencias de las malformaciones congénitas (MFC) con el fin de pesquisar variaciones de dichas frecuencias y poder hacer prevención primaria de ellas ${ }^{14}$. El ECLAMC a su vez es miembro del International Clearinghouse for Birth Defects Monitoring System (ICBDMS, 1991).

En el ECLAMC participan 114 maternidades de 10 países sudamericanos, entre ellos Chile. Ingresan al registro todos los recién nacidos $(\mathrm{RN})$ vivos o mortinatos cuyo peso de nacimiento sea de $500 \mathrm{~g}$ o más. Los RNs son examinados por un pediatra Neonatólogo. A todo $\mathrm{RN}$ que presente una o más MFC se le confecciona una ficha ad-hoc, en la que se especifica el tipo de malformación y además más de 80 datos como fecha de nacimiento, sexo, peso, edad materna y paterna. Se toma como control al niño del mismo sexo, sin malformaciones y que nace a continuación.

En el presente trabajo se estudian los casos registrados en los 15 hospitales chilenos miembros del ECLAMC durante el período de 41 años comprendidos entre 1972-2012.

Las comparaciones estadísticas se hicieron con prueba de $\chi^{2}$ considerando un nivel de significación de 0,05

\section{Resultados}

Durante el período estudiado ocurrieron 798.222 nacimientos. Entre ellos se diagnosticaron 55 RNs portadores de holoprosencefalia en los 15 hospitales chilenos participantes, lo que representa una prevalencia de 0,69 por 10.000 nacimientos. El número de pacientes con HPE fue muy variable en los distintos hospitales. El Hospital Clínico de la Universidad de Chile registró 16 casos, Higueras de Talcahuano 7 casos, San Borja Arriarán de
Santiago 6 y Sótero del Río de Santiago y Hospital de Rancagua 4 casos. Los demás lo hicieron con menor número de casos. Cuarenta y nueve pacientes nacieron vivos y 6 mortinatos. De los pacientes nacidos vivos 13 se fueron de alta $(25,5 \%)$. No sabemos que aconteció posteriormente con ellos. Veintiséis fallecieron $(53,1 \%)$. Hubo 6 pacientes que al momento de cerrar la información figuraban como "sin alta aún". Tampoco tenemos conocimiento de su evolución, ya que no tenemos servicio de seguimiento de estos pacientes.

Treinta y dos RN fueron de sexo masculino $(58,2 \%)$ y 23 femeninos $(41,8 \%)(p>0,05)$. El promedio de edad materna fue de 27,29 años con un rango entre 15 y 41 años. 18,2\% de las madres eran menores de 20 años y 25,5\% mayores de 34 años. El promedio de peso de nacimiento fue de $2.299,4 \mathrm{~g}$ con un rango entre 540 y 4.270 g 56,4\% eran de peso de nacimiento menor de $2.500 \mathrm{~g}$ (Tabla 1).

Como HPE es una anomalía de baja frecuencia, menos de uno por diez mil nacimientos, agrupamos los casos por décadas (Tabla 2). Las tasas de prevalencia al nacimiento de la HPE se mantuvieron relativamente estables a lo largo de los 41 años de observación al comparar las diferentes décadas, no presentando variaciones significativas $\left(\chi^{2}=4,76 ; \mathrm{p}=0,31\right)$.

$56,4 \%$ de los pacientes presentaban defectos craneofaciales. La anomalía más frecuente encontrada en nuestra muestra fue labio leporino medial con paladar hendido (15 casos 27,3\%). Labio

Tabla 1. Factores demográficos presentes en los 55 casos de holoprosencefalia en Chile. Período 1972-2012

\begin{tabular}{|lcc|}
\hline Factores & n & \% \\
\hline Nacidos vivos & 49 & 89,1 \\
Mortinatos & 6 & 10,9 \\
\hline Masculinos & 32 & 58,2 \\
\hline Femeninos & 23 & 41,8 \\
\hline Promedio peso nacimiento en g & $2.299,4$ & \\
\hline Peso menor de 2.500 g & 31 & 56,4 \\
\hline Promedio edad Materna años & 27,29 & \\
\hline Madres menores de 20 años & 10 & 18,2 \\
\hline Madres mayores de 34 años & 14 & 25,5 \\
\hline Madres entre 20 y 35 años & 31 & 56,3 \\
\hline
\end{tabular}


Tabla 2. Número de casos por decenios en el período 1970-2013. Total de nacimientos del período: 798.222. Tasa por 10.000 nacimientos

\begin{tabular}{|cccc|}
\hline Década & Casos & Nacimientos & Tasa \\
\hline $72-79$ & 6 & 84.620 & 0,71 \\
$80-89$ & 5 & 141.991 & 0,55 \\
\hline $90-99$ & 13 & 185.380 & 0,70 \\
\hline $00-09$ & 29 & 332.344 & 0,87 \\
\hline $10-13$ & 2 & 53.887 & 0,37 \\
\hline Total & 55 & 798.222 & 0,69 \\
\hline
\end{tabular}

leporino bilateral sin paladar hendido, 6 casos (11\%), entre ambos 21 casos $(38,2 \%)$. Le siguen 8 casos de Ciclopia (14,5\%), Narina única 6 casos (10,9\%). Cinco casos con Proboscis (9,1\%). Otros defectos específicos craneofaciales se muestran en la Tabla 3.

En $43,6 \%$ de los pacientes se diagnosticaron presencia de malformaciones en otros órganos, no craneofaciales (Tabla 4), siendo las más frecuentes las cardiopatías congénitas $(18,1 \%)$, le siguen en frecuencia polidactilia $(10,9 \%)$, malformaciones renales $(7,3 \%)$ y arteria umbilical única $(5,5 \%)$.

Seis recién nacidos presentaban Trisomía 13 $(10,9 \%)$ y un paciente Trisomía 18 (1,8\%), es decir, $12,7 \%$ de los niños con HPE presentaban una cromosomopatía.

El diagnóstico prenatal se hizo en 30 casos y 9 después del nacimiento. Cabe hacer notar que el antecedente de la edad de diagnóstico no se consignaba en la ficha hasta 1994, lo que ocurrió en 16 pacientes.

\section{Discusión}

Las tasas de prevalencia al nacimiento de HPE publicadas por diferentes autores dan cifras muy variables. La tasa encontrada por nosotros en Chile $(0,69$ por 10 mil nacimientos) es significativamente menor que aquellas consignadas en casuísticas similares, que como la nuestra, incluyen nacidos vivos y mortinatos. Sauders et al. (1984) encontró 1,01 por 10 mil $^{15}$; Croen et al. en 1996: 1,20 por 10 mil ${ }^{16}$; Rasmussen et al. en 19960,86 por $10 \mathrm{mil}^{17}$, sin embargo, otros autores comunican cifras parecidas a las nuestras: 0,72 por 10 mil Witheford
Tabla 3. Defectos craneofaciales específicos encontrados en los 55 casos de holoprosencefalia

\begin{tabular}{|lcc|}
\hline Defecto específico & n & \% \\
\hline Labio y paladar hendido medial & 21 & $38,2 \%$ \\
\hline Ciclopia & 8 & $14,6 \%$ \\
\hline Hidrocefalia & 8 & $14,6 \%$ \\
\hline Microtia con atresia CAE y otras & & \\
\hline Anomalías de la oreja & 7 & $12,7 \%$ \\
\hline Microcefalia & 7 & $12,7 \%$ \\
\hline Narina única & 6 & $10,9 \%$ \\
\hline Proboscis & 5 & $9,1 \%$ \\
\hline Anencefalia & 5 & $9,1 \%$ \\
\hline Hipoplasia nasal & 4 & $7,3 \%$ \\
\hline Defectos craneales & 3 & $5,5 \%$ \\
\hline Microftalmía & 3 & $53,5 \%$ \\
\hline Arrinia & 2 & $3,6 \%$ \\
\hline Micrognatia & 2 & $3,6 \%$ \\
\hline Anoftalmía & 1 & $1,8 \%$ \\
\hline Cefalocele & 1 & $1,8 \%$ \\
\hline Hidranencefalia & 1 & $1,8 \%$ \\
\hline Agenesia del cuerpo calloso & 1 & $1,8 \%$ \\
\hline
\end{tabular}

Tabla 4. Defectos no craneofaciales encontrados en los 55 casos de holoprosencefalia

\begin{tabular}{|lcc|}
\hline Defecto específico & $\mathbf{n}$ & $\mathbf{\%}$ \\
\hline Cardiopatía congénita & 10 & $18,2 \%$ \\
\hline Polidactilia & 6 & $10,9 \%$ \\
\hline Malformaciones renales & 4 & $7,3 \%$ \\
\hline Arteria umbical única & 3 & $5,5 \%$ \\
Hipoplasia pulmonar & 2 & $3,6 \%$ \\
Hernia diafragmática & 1 & $1,8 \%$ \\
\hline Onfalocele & 1 & $1,8 \%$ \\
\hline
\end{tabular}

y Tolmie $1996^{18}$ y 0,77 por 10 mil Mastroiacobo et al. $1992^{9}$.

Hubo una amplia variabilidad clínica entre los pacientes estudiados, como era de esperar, en el espectro de la HPE.

El discreto aumento observado por nosotros de las tasas entre el período 1972-1979 (0,71 
por 10.000) y el decenio 2000-2009 (0,87 por 10.000), no alcanza significación estadística, y se debería probablemente a la introducción masiva de exámenes prenatales como la ecografía, tanto prenatal como postnatal lo que explicaría la mayor concentración de casos en los hospitales de grandes ciudades como Santiago, Rancagua, Concepción, que cuentan con una tecnología más avanzada. Por el contrario, es muy probable que la baja frecuencia encontrada en Chile se pueda deber a un subregistro en alguno o varios hospitales participantes.

La prevalencia de la holoprosencefalia en niños de sexo masculino fue más frecuente que el femenino (razón de sexos $\mathrm{M} / \mathrm{F}=1,4$ ), lo cual es diferente a lo encontrado por Orioli y Castilla ${ }^{20}$ : 0,74, pero ello podría deberse al azar debido al pequeño tamaño de nuestra muestra.

Mastroiacobo et al. ${ }^{19}$ consideran que Labio Leporino es parte del espectro de la HPE, lo que apoya nuestros hallazgos. También Mastroiacobo et al. ${ }^{19}$ y Orioli y Castilla ${ }^{20}$ afirman que los defectos severos de los oídos, con atresia del conducto auditivo externo, forman parte del espectro de defectos de la HPE, lo mismo que la microstomía, lo que también confirman lo encontrado en nuestra muestra. En opinión de Cohen ${ }^{21,22}$ la fisura labial medial con perímetro cefálico normal rara vez corresponde a una HPE subyacente. Generalmente, una HPE con fisura labial medial con circunferencia cefálica normal tiene mejor pronóstico que si estuviera asociada a microcefalia. Le siguen en frecuencia Ciclopia 14,5\%, Narina única $10,9 \%$, Proboscis $9,1 \%$. Otros defectos específicos craneofaciales se muestran en la Tabla 4. Hubo 7 casos de Microcefalia (12,7\%) y 5 de anencefalia $(9,1 \%)$.

Cohen et al. ${ }^{22,23}$ comprobaron la asociación de HPE con anencefalia, espina bífida y polidactilia. Esta asociación la encontramos también en nuestro estudio.

El hecho que el 10,9\% de recién nacidos con HPE presentaran Trisomía 13 y 1,8\% Trisomía 18 ha sido comunicado por varios autores (Mastroiacobo et al. ${ }^{19}$, Orioli y Castilla ${ }^{20}$, Chen et al. ${ }^{23}$ ).

Según De $\mathrm{Myer}^{8}$ el grado de gravedad de la HPE se puede predecir a partir de los defectos de la línea medio-facial que forman un espectro desde el mayor al menor grado de compromiso de la enfermedad. Asegura que en todos los casos, sin excepción, los siguientes signos, cuando están presentes, son indicadores de HPE Alobar: grado variable de compromiso del cerebro, Ciclopia, Etmocefalia, y Cebocefalia. Labio leporino bilateral o unilateral con compromiso de la premaxila y del prolabio son propios de la HPE semilobar o lobar. El labio leporino central puede presentarse en los tres tipos de HPE.

Es muy importante el examen físico dirigido a detectar signos clínicos menores en los casos de holoprosencefalia lobar, ya que muchas veces el diagnóstico se debe sospechar en pacientes que presentan signos leves que pueden no ser interpretados adecuadamente, como ausencia del frenillo bajo el labio superior, nariz aplanada e incisivo central superior único en niños mayores.

Es también relevante precisar el tipo de HPE y el grado de compromiso cerebral ya que ello nos ayuda a hacer un pronóstico del desarrollo psicomotor del paciente y la indicación del tratamiento quirúrgico de los defectos faciales.

\section{Conclusión}

La Holoprosencefalia en Chile, es una enfermedad rara, con una prevalencia al nacimiento de 0,69 por diez mil, según los datos del ECLAMC, menor que en numerosos otros países. Los signos clínicos externos craneofaciales más frecuentes que encontramos son: Labio leporino medial con o sin paladar hendido, ciclopia, proboscis y narina única. Las asociaciones más frecuentes con patologías en otros órganos son cardiopatías congénitas, espina bífida y polidactilia. 10,9\% de los casos tenían como patología de base una Trisomía 13. No ha habido en Chile un aumento de la prevalencia significativa a lo largo de los 41 años de seguimiento de este estudio.

\section{Referencias}

1. Cohen MM, Sulik KK. Perspectives on holoprosencephaly: Part II. Central Nervous system, craniofacial anatomy, sybdrome, commentary, diagnostic approach and experimental studies. J Craneofac Genet Dev Biol 1992; 12: 196-244.

2. Matsunaga GE, Shiota K. Holoprosencephaly in human embrios: Epidemiologic studies of 150 cases. Teratologic 1977; 15 (3): 261-72.

3. Orioli IM, Castilla EE. Epidemiology of Holoprosencephaly: Prevalence and risk factors. Am J Med Genet 
Part C (seminars in Medical Genetics) 2010: 154C: 1321.

4. Orioli IM, Castilla EE, Ming JE, Nazer J, Burle de Agiar $\mathrm{M}$, Llerena JC, et al. Identification of novel mutations in SHH and ZIC2 in South American (ECLAMC), population with holprosencephaly. Hum Genet 2001; 109: 1-6.

5. Berry SM, Gosden C, Snidjers RJM. Fetal holoprosencephaly: Associated malformations and chromosomal defects. Fetal Diagn Ther 1990; 5: 92-9.

6. De Myer W, Zeman W, Palmer GP. Alobar prosencephaly (Arrhinencephaly) whith median cleft lip and palate: clinical, electroencephalografic and nosologic considerations. Confin Neurol 1963; 23: 1-36.

7. De Myer W, Zeman W. The median Cleft Face Syndrome. Neurol 1967; 17: 961-71.

8. Cohen MM, Shiota K. Teratogenesis of holoprosencephaly. Am J of Medical Genet 2002; 109 (1): 1-15.

9. De Myer W, Zeman W, Palmer CG. The face predicts the brain: Diagnostic significance of median facial anomalies for holoprosencephaly (Arrhinencephaly). Pediatrics 1964; 34: 256-63.

10. Nelson LH, King M. Early diagnosis of holoprosencephaly. J Ultrasound Med 1992; 11: 57-9.

11. Filly RA, Chinn DH, Callen PW. Alobar prosencephaly: Ultrasonographic prenatal diagnosis 1984; 151: 455-9.

12. McGahan JP, Nyberg DA, Mack LA. Sonography of facial features of alobar and semilobar holoprosencephaly. AJR Am J Roentgeno 1990; 154: 143-8.

13. Castilla EE, Orioli IM. The Latin American Collaborative Study of Congenital Malformations. Community Genet 2004; 7 (2-3): 338-45.

14. Saunders ES, Shortland D, Dunn PM. What is the incidence of holoprosencephaly? J Med Genet 1984; 21: 21-6.
15. Croen LA, Shaw GM, Lammer EJ. Holoprosencephaly Epidemiologic and clinical Characteristics of a California population. Am J Med Genet 1996; 64: 465-72.

16. Rasmusen SA, Moore CA, Khloury MJ, Cordero JF. Descriptive epidemiology of holoprosencephaly and arhinencefaly in Metropolitan Atlanta, 1968-1992. Am J Med Genet 1996; 66: 320-33.

17. Whiteford ML, Tolmie JL. Holoprosencephaly in the West of Scotland 1975-1994. J Med Genet 1996; 33: 578-84.

18. Mastroiacobo P, Botto LD, Cavalcanti DP, Zampino G, Serafini MA. Epidemiological and genetic study of holoprosencephaly in 206 cases observed in the Italian Multicentric Registry 1978-1989. Paper presented at proceedings of the $1^{\text {st }}$ international meeting of the genetic reproductive epidemiology Research Society (GRERS). Roma 1992.

19. Orioli IM, Castilla E. Clinical Epidemiologic Study of Holoprosencephaly in South America. Am J Med Genet Part A 2007; 143A: 3088-99.

20. Cohen MM Jr. Problems in the definition of holoprosencephaly. Am J Med Genet 2001; 103: 183-7.

21. Cohen MM Jr. Perspectives on holoprosencephaly: Part I. Epidemiology, genetics, and sindromology. Teratology. 1989a; 40: 211-35.

22. Cohen Jr. Perspectives on holoprosencephaly: Part III. Spectra, distinctions, continuities, and discontinuities. Am J Med Genet 1989b; 34: 271-88.

23. Chen CP, Chern SR, Lin CJ, Lee CC,Wang W, Tzen CY. A comparison of maternal age, sex ratio and associated anomalies among numerically aneuploid, structurally aneuploid and euploid holoprosencephaly. Genet Council 2005 ; 16: 49-57. 